\title{
Influence of season, harvest time and drying on Java citronella (Cymbopogon winterianus Jowitt) volatile oil
}

\author{
Arie F. Blank ${ }^{1 *}$, Andressa G. Costa ${ }^{1}$, Maria de Fátima Arrigoni-Blank ${ }^{2}$, Sócrates C. H. \\ Cavalcanti ${ }^{3}$ Péricles B. Alves ${ }^{4}$, Renato Innecco ${ }^{5}$, Polyana A. D. Ehlert ${ }^{\text {, }}$ \\ Inajá Francisco de Sousa ${ }^{1}$ \\ ${ }^{1}$ Departamento de Engenharia Agronômica, Universidade Federal de Sergipe, Campus Prof. José Aloisio de \\ Campos, Av. Marechal Rondon s/n, 49100-000, São Cristóvão, SE, Brazil, \\ ${ }^{2}$ Departamento de Biologia, Universidade Federal de Sergipe, Campus Prof. Alberto Carvalho, \\ Av. Vereador Olímpio Grande s/n, 49500-000, Itabaiana, SE, Brazil, \\ ${ }^{3}$ Departamento de Fisiologia, Universidade Federal de Sergipe, Campus Prof. José Aloísio de Campos, Av. \\ Marechal Rondon s/n, 49100-000, São Cristóvão, SE, Brazil, \\ ${ }^{4}$ Departamento de Química, Universidade Federal de Sergipe, Campus Prof. José Aloísio de Campos, Av. Marechal \\ Rondon s/n, 49100-000, São Cristóvão, SE, Brazil, \\ ${ }^{5}$ Departamento de Fitotecnia, Universidade Federal do Ceará, Caixa-Postal 12168, Campus \\ do Pici, 60356-001, Fortaleza, CE, Brazil
}

\begin{abstract}
RESUMO: "Influência da estação, do horário de colheita e da secagem no óleo essencial de citronela de java (Cymbopogon winterianus Jowitt)". Citronela de Java (Cymbopogon winterianus Jowitt) pertence à família Poaceae. Óleo volátil de citronela de Java apresenta atividade repelente, antimicrobiana e acaricida. Sabe-se que fatores agronômicos têm grande efeito sobre a qualidade e quantidade de metabólitos essenciais. Por isso é importante a determinação dos níveis ótimos dos fatores agronômicos que afetem o crescimento e a produção. Horário de colheita e secagem são fatores agronômicos muito importantes. O presente estudo foi conduzido na Fazenda Experimental do Departamento de Engenharia Agronômica da Universidade Federal de Sergipe, no período de 2002-2003, usando o delineamento de blocos casualizados e um experimento em esquema fatorial com três repetições. O capim citronela foi cultivado usando espaçamento de $60 \mathrm{~cm}$ entre linhas e $60 \mathrm{~cm}$ entre plantas. Realizaram-se colheitas de manhã, meio dia e à tarde, às 9:00 h, 12:00 h e 15:00 h, nas quatro estações do ano. Usaram-se folhas frescas e secas no experimento. Para estudar os efeitos do horário de colheita e secagem avaliaram-se as seguintes características: rendimento de biomassa seca e fresca $(\mathrm{kg} / \mathrm{ha})$, umidade $(\%)$, teor $(\%)$ e rendimento (L/ha) de óleo essencial, e a composição química do óleo essencial. Mudanças sazonais apresentaram efeito significativo sobre o rendimento de biomassa fresca e seca, e teor e rendimento de óleo. Rendimentos máximos de óleo essencial foram observados às 9:00 h durante o verão, inverno e primavera. $O$ teor de óleo essencial foi influenciado pela estação doa ano e secagem, mas não foi influenciado pelo horário de colheita.
\end{abstract}

Unitermos: Cymbopogon winterianus, colheita, pós-colheita, geraniol, citronelal, citronelol.

\begin{abstract}
Java citronella (Cymbopogon winterianus Jowitt) is member of the Poaceae family. Java citronella volatile oil has been reported to be among the volatile oils, showing repellent, antimycotic, and acaricide activities. It has been known that agronomical factors have a great effect on both the quality and quantity of essential metabolites. For this reason, it is necessary to determine optimum levels of agronomical factors affecting plant growth and production. Harvest time and drying are very important agronomical factors. This study has been conducted in the Research farm of the "Universidade Federal de Sergipe", Agronomical Engineering Department along 2002-2003 on the base of factorial experiment in randomized complete block design with three replications. Java citronella was cultivated in a $60 \times 60 \mathrm{~cm}$ space. Early, midday, and late harvest at 9:00 h, 12:00 h, and 15:00 h were conducted on four different seasons. Fresh and dried leaves were used on the experiments. In order to study the effects of harvest time and drying, yields of dry and fresh herbage (kg/ha), moisture content (\%), volatile oil content (\%) and yield (L/ ha), and chemical composition of the volatile oil were measured. Seasonal changes had significant effect on yield of fresh herbage, yield and volatile oil content. Maximum volatile oil yields were observed at 9:00 during summer, winter, and spring. Volatile oil content was influenced by season and drying, but not influenced by harvest time.
\end{abstract}

Keywords: Cymbopogon winterianus, harvest, post-harvest, geraniol, citronellal, citronellol. 


\section{INTRODUCTION}

The Cymbopogon genus is composed of more than 100 species found in tropical countries (Lorenzi; Matos et al., 2003). About 56 species are aromatic and some of them of medicinal, pharmacological and industrial importance (Morais et al., 2005; Tôrres et al., 2005; Vendruscolo et al., 2005; Barbosa-Filho et al., 2005; Singi et al., 2005; Pansera et al., 2003). Two species of Cymbopogon are known according to their region. $C$. nardus (Jamarosa) and C. winterianus (Java citronella) have similar volatile oil scent and medicinal uses, but show different citronellal content. Java citronella is rich in geraniol $(36.0 \%)$ and citronellal $(42.7 \%)$ and shows repellent, antimycotic, and acaricide activities. It is reported to be an air freshener (Guenther, 1992; Matos, 2000).

The biosynthesis of secondary metabolites, although controlled genetically, is strongly affected by environmental, harvest and post-harvest factors. Agricultural factors have a critical effect on quantitative and qualitative characteristics of Java citronella, that affect plant growth and yield. Precipitation, temperature, light, and humidity influence volatile oil yield and main constituents content (citronellol, citronellal, and geraniol) (Sarma, 2002). Sarma et al. (2001) cultivated Java citronella between 1998 and 2000 in India northeast. They found a large variation on volatile oil yield and content depending on seasonal changes and harvesting time. Highest yields and citronellal content were obtained during September and October. Under drought, the geraniol and citronellal contents were reduced, whereas citronellol increased (Fatima et al., 2002).

Java citronella cultivated at an altitude of 60 $\mathrm{m}$ showed elevated biomass production $(24.5 \mathrm{t} / \mathrm{ha})$ and volatile oil content $(1.3 \%)$. An increase in citronellal content and a decrease in citronellol and geraniol were observed at this altitude (Sarma et al., 2001). However, no studies had been conducted on the effects of harvest time and drying during the four seasons in the northeast region of Brazil. As a part of our ongoing work on the evaluation of agricultural factors influencing the quality and quantity of volatile oil constituents (Arrigoni-Blank et al., 2005; Carvalho-Filho et al., 2006), we report on the results obtained for $C$. winterianus to study the influences of harvest time and drying throughout different seasons.

\section{MATERIAL AND METHODS}

This experiment has been conducted under rainfed condition at the Research farm of the "Universidade Federal de Sergipe", Department of Agronomical Engineering, São Cristóvão, State of Sergipe, Brazil $\left(10^{\circ} \mathrm{C} 55^{\prime} \mathrm{S}, 37^{\circ} \mathrm{C} 11^{\prime} \mathrm{W}, 20 \mathrm{~m}\right.$ above sea level) between 12 December 2002 and 12 November 2003. Weather data is shown in Figures 1 and 2. No fertilization procedures have been carried out.
We transplanted the tillers into the experimental field on 12 May 2002, using a $60 \times 60 \mathrm{~cm}$ space. The experiment design was a factorial with three replications.

Early (9:00 h), midday (12:00 h), and late (15:00 h) harvest were performed in four different seasons (summer, 12 Dec. 2002; fall, 02 Apr. 2003; winter, 21 Aug. 2003, and spring, 12 Nov. 2003). We used fresh and dry leaves for volatile oil distillation. In order to study the effects of harvest time and drying, yields of dry and fresh herbage $(\mathrm{kg} / \mathrm{ha})$, moisture content $(\%)$, volatile oil content $(\%)$ and yield $(\mathrm{L} / \mathrm{ha})$, and chemical composition of the volatile oil were measured.

The experimental unit consisted of two plants. Plants were cut $20 \mathrm{~cm}$ above soil level. The volatile oils of leaves were extracted by hydrodistillation for 3 h (Carvalho-Filho et al., 2006), using a Clevenger-type apparatus on a 3 liter flask (British Pharmacopoeia, 1988). The volatile oils were separated from the aqueous phase and kept in the freezer $\left(-20^{\circ} \mathrm{C}\right)$ until further use. Volatile oil contents were estimated based on dry herbage weight using three $200 \mathrm{~g}$ samples of fresh herbage or $75 \mathrm{~g}$ of dry herbage (American Spice Trade Association, 1985). Dried leaves were obtained by drying at $40{ }^{\circ} \mathrm{C}$ in a forced air oven (Marconi) for 5 days.

Qualitative and quantitative analyses of volatile oils were performed with a Shimadzu QP5050A GC/MS equipped with a DB-5MS fused silica column (30 m x $0.25 \mathrm{~mm}$; film thickness $0.25 \mu \mathrm{M}$ ), under the following conditions: helium as carrier gas at $1.0 \mathrm{~mL} / \mathrm{min}$; injector split at $250^{\circ} \mathrm{C}$ (split ratio $\left.1 / 20\right)$; detector at $280^{\circ} \mathrm{C}$, column temperature program $80{ }^{\circ} \mathrm{C}$ during $1.5 \mathrm{~min}$, with $4{ }^{\circ} \mathrm{C}$ increases per minute to $180{ }^{\circ} \mathrm{C}$, then $10{ }^{\circ} \mathrm{C} / \mathrm{min}$ to 300 ${ }^{\circ} \mathrm{C}$, ending with a $10 \mathrm{~min}$ isothermal at $300^{\circ} \mathrm{C}$. The mass spectra were taken at $70 \mathrm{eV}$ with scanning speed of 0.85 $\mathrm{scan} / \mathrm{s}$ from 40 to $550 \mathrm{Da}$. Constituents identification was assigned on basis of comparison of their retention indices relative $n$-alkane homologous series obtained by coinjecting the volatile oil sample with a linear hydrocarbon mixture as well as by computerized matching of the acquired mass spectra with those stored in NIST21 and NIST107 mass spectral library of the GC/MS data system and other published mass spectra (Adams, 1995).

Analysis of variance of the results was conducted using the SISVAR program, and means in the results were compared using the Tukey's test $(\mathrm{P}<0.05)$.

\section{RESULTS AND DISCUSSION}

The Tukey test comparing treatments are shown in Tables 1 and 2 . Highest fresh biomass resulted from summer $(9,326 \mathrm{~kg} / \mathrm{ha})$, fall $(8,174 \mathrm{~kg} / \mathrm{ha})$, and spring $(8,352 \mathrm{~kg} / \mathrm{ha})($ Table 1$)$ and the lowest one resulted from winter $(3,788 \mathrm{~kg} / \mathrm{ha})$. Season had significant effects on dry herbage biomass. Higher dry biomass production was achieved from fall and summer $(5,363 \mathrm{~kg} / \mathrm{ha}$ and 4,897 $\mathrm{kg} / \mathrm{ha}$, respectively). The lower dry biomass production 
resulted from winter and spring $(1,625 \mathrm{~kg} / \mathrm{ha}$ and $3,189 \mathrm{~kg} /$ ha, respectively), however proportionally higher moisture was observed $(61.82 \%$ and $57.11 \%$, respectively; Table 1). This is due to higher pluviometric readings during both seasons, contributing to higher biomass moisture, in addition to the plant stage immediately after blooming during harvest, when leaves were very young. However, Innecco et al. (2003) did not observe this result, studying Mentha $x$ vilosa Huds, probably because irrigation was performed during dry seasons. Higher fresh and dry biomasses also were observed during dry seasons with irrigation.

Another important factor influencing Java citronella volatile oil production is harvesting time. Most volatile oils were produced at 9:00 h $(2.71,2.36$, and $4.24 \%$ for summer, winter, and spring seasons, respectively), because volatile oil content was generally found to peak at that time, except during fall, when there were no significant differences between harvest times.

The content ( $\%)$ of volatile oil in dried herbage was not significantly influenced by harvesting time. However, higher contents of volatile oil were obtained during spring $(4.24 \%$, fresh biomass and $3.40 \%$, dry biomass) and fall (3.17\%, dry biomass).

Harvest at 12:00 $\mathrm{h}$ resulted in quite similar volatile oil content throughout all seasons. In general 15:00 h harvest significantly reduced volatile oil content, showing the lowest value during winter season $(2.33 \%$ dry biomass), characterized by higher precipitation volumes (Table 2).

Similar results have been reported (Nascimento et al., 2003; Nagao et al., 2004). In accordance with studies on Cymbopogon citratus (DC) Stapf conducted by Nascimento et al. (2003), harvest performed at 9:00 and 11:00 h, providing higher volatile oil content $(5.59$ and $5.31 \%$, respectively). The chemotype citral/limonene of Lippia alba (Mill) N.E.Br. produces lower volatile oil content during the rainy season, which accounts for a potential metabolic decrease caused by reduction on solar radiation (Nagao et al., 2004).

In general winter harvest significantly reduced yield (109.1 L/ha, average) independently of harvest time (Table 2). Increased pluvial readings and the beginning of plants vegetative period may have accounted for these results.

Drying significantly increased volatile oil

Table 1. Effects of season on Java citronella (Cymbopogon winterianus Jowitt) fresh and dry biomass and moisture.*

\begin{tabular}{lccc}
\hline \multicolumn{1}{c}{ Season } & Fresh biomass yield $(\mathrm{kg} / \mathrm{ha})$ & Dry biomass yield $(\mathrm{kg} / \mathrm{ha})$ & Moisture content $(\%)$ \\
\hline Summer & $9,326 \mathrm{a}$ & $4,897 \mathrm{a}$ & 34.4 \\
Fall & $8,174 \mathrm{a}$ & $5,363 \mathrm{a}$ & 47.5 \\
Winter & $3,788 \mathrm{~b}$ & $1,625 \mathrm{c}$ & 61.8 \\
Spring & $8,352 \mathrm{a}$ & $3,189 \mathrm{~b}$ & 57.1 \\
\hline
\end{tabular}

* Means in each column followed by the same letter are not significantly different $(\mathrm{P}<0.05)$.

Table 2. Influence of seasons and harvest times on volatile oil content (\%) from fresh and dry leaves of C. winterianus.*

\begin{tabular}{lcccccc}
\hline \multirow{2}{*}{ Season } & \multicolumn{3}{c}{ Oil content (\%) } & \multicolumn{2}{c}{ Oil yield (L/ha) } \\
\cline { 2 - 7 } & $9: 00 \mathrm{~h}$ & $12: 00 \mathrm{~h}$ & $15: 00 \mathrm{~h}$ & $9: 00 \mathrm{~h}$ & $12: 00 \mathrm{~h}$ & $15: 00 \mathrm{~h}$ \\
\cline { 2 - 7 } & \multicolumn{5}{c}{ Fresh leaves } \\
\hline Summer & $2.71 \mathrm{~b} \mathrm{~A}$ & $2.35 \mathrm{~b} \mathrm{AB}$ & $1.98 \mathrm{a} \mathrm{B}$ & $162.50 \mathrm{a}$ & $114.82 \mathrm{a}$ & $97.17 \mathrm{a}$ \\
Fall & $2.22 \mathrm{~b} \mathrm{~A}$ & $1.84 \mathrm{~b} \mathrm{~A}$ & $1.71 \mathrm{a} \mathrm{A}$ & $119.10 \mathrm{a}$ & $91.47 \mathrm{a}$ & $98.46 \mathrm{a}$ \\
Winter & $2.36 \mathrm{~b} \mathrm{~A}$ & $1.98 \mathrm{~b} \mathrm{AB}$ & $1.67 \mathrm{a} \mathrm{B}$ & $38.39 \mathrm{~b}$ & $32.13 \mathrm{~b}$ & $27.13 \mathrm{~b}$ \\
Spring & $4.24 \mathrm{a} \mathrm{A}$ & $3.36 \mathrm{a} \mathrm{B}$ & $2.43 \mathrm{a} \mathrm{C}$ & $135.29 \mathrm{a}$ & $107.25 \mathrm{a}$ & $77.42 \mathrm{a}$ \\
\hline & & & Dry leaves & & $151.80 \mathrm{a}$ \\
\hline Summer & $2.60 \mathrm{~b} \mathrm{~A}$ & $2.47 \mathrm{a} \mathrm{A}$ & $3.10 \mathrm{a} \mathrm{A}$ & $127.32 \mathrm{~b}$ & $120.79 \mathrm{~b}$ & $171.61 \mathrm{a}$ \\
Fall & $3.17 \mathrm{ab} \mathrm{A}$ & $3.00 \mathrm{a} \mathrm{A}$ & $3.20 \mathrm{a} \mathrm{A}$ & $169.82 \mathrm{a}$ & $160.88 \mathrm{a}$ & $37.91 \mathrm{c}$ \\
Winter & $2.57 \mathrm{~b} \mathrm{~A}$ & $2.67 \mathrm{a} \mathrm{A}$ & $2.33 \mathrm{~b} \mathrm{~A}$ & $41.70 \mathrm{c}$ & $43.32 \mathrm{c}$ & $111.62 \mathrm{~b}$ \\
Spring & $3.40 \mathrm{a} \mathrm{A}$ & $3.20 \mathrm{a} \mathrm{A}$ & $3.50 \mathrm{a} \mathrm{A}$ & $108.43 \mathrm{~b}$ & $102.05 \mathrm{~b}$ & \\
\hline
\end{tabular}

* Means followed by the same minuscule letter in each column and by the same capital letter in each line are not significantly 
content and yield, this is probably due to the drying process affecting cell membrane resistance, assisting the volatile oil release during hydrodistillation. However, other species, such as, Ocimum basilicum L. did not show significant differences between fresh and dry biomass (Carvalho-Filho et al., 2006) and Lippia alba showed peak volatile oil content at 15:00 $\mathrm{h}$ in both rainy and dry seasons. Specifically during the rainy season, volatile oil content increased throughout midday decreasing by 17:00 h. Daily variations in temperature and luminosity may account for these results.

The major components in Cymbopogon winterianus volatile oil were identified as limonene, citronellal, citronellol, neral, geraniol, geranial, and farnesol (Tables 3 and 4).

The GC/MS results showed that geraniol is an important volatile constituent in the volatile oil of
Cymbopogon winterianus, as well as citronellal. The content of these two compounds in fresh biomass varied with the season. However, no significant variation was observed for dry biomass. In the rainy period (winter), the citronellal in fresh biomass reached the lowest values at 9:00 $\mathrm{h}$ and 12:00 $\mathrm{h}$, while geraniol content increased.

However, in the dry period (summer) the content of citronellal increased to $30.54 \%$ at $15: 00$, while the content of geraniol decreased to its lowest value $(21.78 \%)$. Diverging results were observed by Rocha et al. (2002), maximum production of citronellal was found between 9:00 and 11:00 $\mathrm{h}$.

The least value of limonene $(1.17 \%)$ was observed in fresh leaves, during fall at 12:00. However, the highest limonene yield and content was achieved in plants harvested during winter.

The content of citronellol in fresh biomass
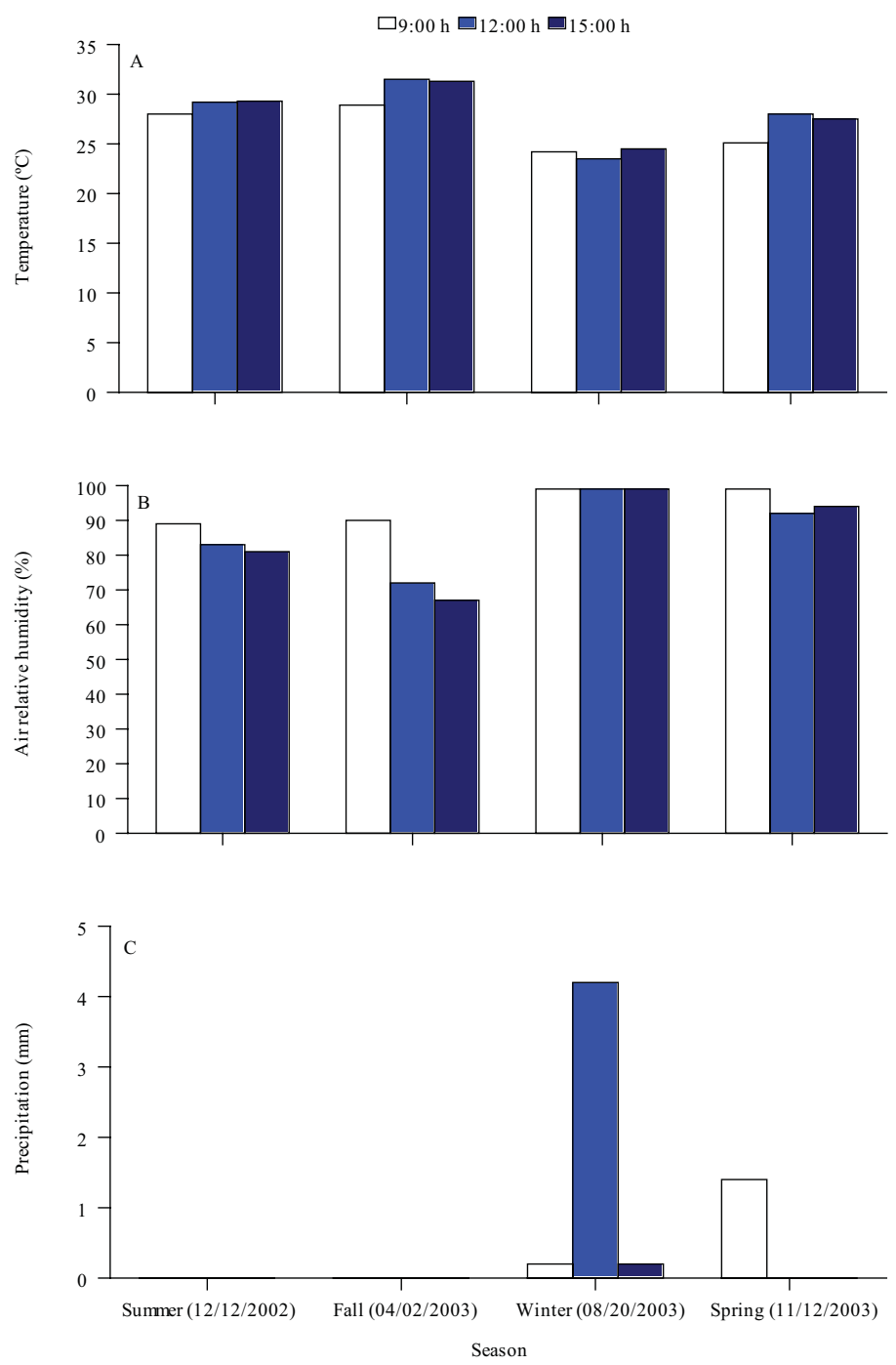

Figure 1. Temperature $\left({ }^{\circ} \mathrm{C}\right)(\mathrm{A})$, air relative humidity $(\%)(\mathrm{B})$ and precipitation $(\mathrm{mm})(\mathrm{C})$ in the Research farm of UFS during harvest. 
significantly decreased at 15:00 $\mathrm{h}$ during winter. The content of citronellol in dry biomass had no significant variation (Tables 3 and 4). Drying provided an increase in citronellol during summer at 12:00 $\mathrm{h}$, while in the rainy period a reduction was observed at 9:00 and 12:00 h.

In general, no significant differences in the content of neral were found in fresh and dry biomass throughout the harvest time and seasons, except for winter at 9:00, where neral was absent. Higher content of neral was obtained by drying biomass harvested at 9:00 h during winter (Tables 3 and 4). Similarly, the content of neral increased after drying Melissa officinalis $\mathrm{L}$. for five days at $40{ }^{\circ} \mathrm{C}$ (Blank et al., 2005).

Amounts of geranial in fresh and dry biomass were relatively low and not significantly different, except dry herbage harvested at 12:00 during fall (Tables 3 and 4).

Peak farnesol content was achieved only on fresh herbage during winter at 9:00 h, which decreased after drying. After drying, herbage harvested at 9:00 and 12:00 during summer showed highest farnesol content (5.69\% and $6.13 \%$, respectively; Tables 3 and 4).

According to Sarma (2002), Java citronella showed better results in high precipitation (100-200 $\mathrm{mm}$ ), temperatures between $20{ }^{\circ} \mathrm{C}$ and $30{ }^{\circ} \mathrm{C}$, and high moisture. In India, citronellal is in higher concentration during September and October, period with favorable conditions locally. Such conditions were found during our experiments, favoring the achieved results.

Season affected chemical composition significantly. Time of harvest had little influence on composition. Drying reduced limonene, citronellol, geraniol, and farnesol and increased citronellal and neral contents. Similar results were obtained for Melissa officinalis. Drying reduced nerol and geraniol contents to trace amounts (Blank et al., 2005).
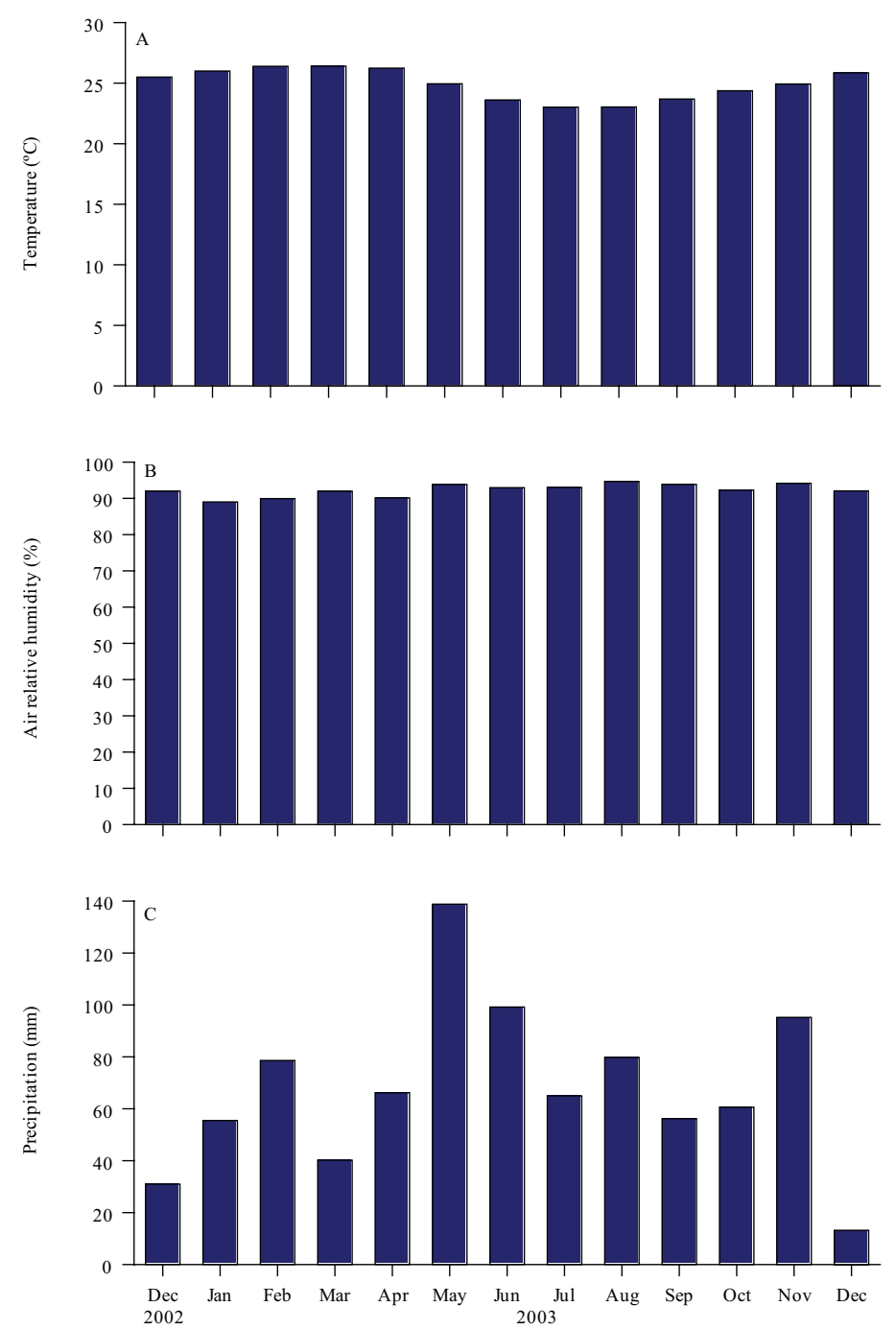

Figure 2. Monthly temperature averages $\left({ }^{\circ} \mathrm{C}\right)(\mathrm{A})$, air relative humidity $(\%)(\mathrm{B})$, and pluvial precipitation $(\mathrm{mm})(\mathrm{C})$ in the Research farm of UFS. 
Table 3. Composition of volatile oil from fresh leaves of Java citronella (C. winterianus). Relative percentages as a function of seasons and harvest times.

\begin{tabular}{|c|c|c|c|c|c|c|c|c|c|c|c|c|}
\hline \multirow{2}{*}{ Compounds } & \multicolumn{3}{|c|}{ Summer } & \multicolumn{3}{|c|}{ Fall } & \multicolumn{3}{|c|}{ Winter } & \multicolumn{3}{|c|}{ Spring } \\
\hline & $9: 00 \mathrm{~h}$ & $12: 00 \mathrm{~h}$ & $15: 00 \mathrm{~h}$ & $9: 00 \mathrm{~h}$ & $12: 00 \mathrm{~h}$ & $15: 00 \mathrm{~h}$ & $9: 00 \mathrm{~h}$ & $12: 00 \mathrm{~h}$ & $15: 00 \mathrm{~h}$ & $9: 00 \mathrm{~h}$ & $12: 00 \mathrm{~h}$ & $15: 00 \mathrm{~h}$ \\
\hline & \multicolumn{12}{|c|}{ Relative percentage of chemical constituents } \\
\hline 6-metil-2-hepten-2-one & 0.04 & -- & -- & -- & -- & -- & 0.20 & 0.21 & -- & -- & -- & -- \\
\hline$\beta$-Mirceno & 0.07 & -- & -- & -- & -- & -- & 0.13 & 0.04 & -- & -- & -- & -- \\
\hline Octanal & 0.07 & -- & -- & -- & -- & -- & 0.08 & -- & -- & -- & -- & -- \\
\hline Limoneno & 2.73 & 2.96 & 2.63 & 1.20 & 1.17 & 1.27 & 3.32 & 3.51 & 2.32 & 1.78 & 1.77 & 1.85 \\
\hline a-pinene & 0.34 & 0.38 & 0.32 & 0.07 & 0.08 & 0.13 & 0.42 & 0.37 & 0.27 & 0.10 & 0.28 & 0.21 \\
\hline Trans- $\beta-O c i m e n e$ & 0.21 & 0.15 & 0.38 & -- & -- & -- & 0.26 & 0.22 & 0.16 & 0.04 & -- & -- \\
\hline Bergamal & 0.60 & 0.42 & 0.60 & 0.17 & 0.09 & 0.17 & 0.75 & 0.71 & 0.40 & -- & -- & -- \\
\hline Nonanone & 0.49 & 0.29 & 0.30 & 0.16 & 0.17 & 0.20 & 0.58 & 0.62 & 0.33 & 0.07 & 0.08 & 0.14 \\
\hline Linalol & 0.68 & 0.75 & 0.37 & 1.29 & 1.04 & 1.08 & 1.52 & 2.05 & 1.03 & 1.12 & 1.02 & 1.07 \\
\hline Rose Oxide & 0.13 & -- & 0.17 & -- & -- & -- & 0.25 & 0.12 & 0.04 & -- & -- & -- \\
\hline Isopulegol & 0.03 & 0.37 & 14.89 & 1.14 & 0.73 & 0.77 & 0.03 & 0.17 & 0.24 & 0.79 & 0.66 & 0.67 \\
\hline neo-Isopulegol & 0.25 & -- & 0.06 & -- & -- & -- & -- & 0.10 & 0.34 & -- & -- & -- \\
\hline Citronelal & 26.66 & 21.42 & 30.54 & 22.65 & 24.71 & 25.53 & 0.21 & 0.08 & 26.92 & 28.59 & 26.58 & 27.58 \\
\hline Terpenol-4 & 0.07 & -- & -- & -- & -- & -- & -- & -- & -- & -- & -- & -- \\
\hline cis-Decenal & 0.08 & -- & 0.03 & -- & -- & -- & 0.04 & 0.04 & -- & -- & -- & -- \\
\hline Decanal & 0.81 & 0.64 & 0.75 & 0.46 & 0.46 & 0.44 & 0.95 & 1.01 & 0.57 & 0.45 & 0.47 & 0.45 \\
\hline Citronelol & 10.13 & 10.75 & 8.43 & 11.99 & 11.58 & 12.47 & 26.16 & 27.00 & 14.05 & 12.16 & 11.33 & 11.38 \\
\hline Neral & 10.17 & 4.33 & 5.88 & 4.62 & 4.60 & 5.21 & -- & -- & 4.58 & 4.47 & 3.98 & 4.17 \\
\hline Geraniol & 34.16 & 37.29 & 21.78 & 43.55 & 44.54 & 42.73 & 47.67 & 48.72 & 38.00 & 41.81 & 44.86 & 42.59 \\
\hline Geranial & -- & 0.68 & -- & 3.68 & 1.96 & 2.57 & -- & -- & 0.75 & 3.26 & 1.42 & 1.57 \\
\hline Geranyl Formate & -- & -- & -- & -- & -- & -- & -- & -- & -- & -- & -- & -- \\
\hline Citronelyl acetate & 0.64 & 1.00 & 0.74 & 0.98 & 0.97 & 0.76 & 0.99 & 1.89 & 0.86 & 0.95 & 0.98 & 1.18 \\
\hline Geranyl acetate & 1.06 & 0.74 & 0.86 & 1.57 & 1.82 & 1.37 & 2.04 & 3.14 & 2.36 & 0.93 & 1.78 & 2.37 \\
\hline$\beta$-Elemene & -- & 0.15 & -- & -- & -- & -- & -- & -- & -- & -- & -- & -- \\
\hline Tetradecanal & 1.05 & -- & -- & -- & -- & -- & 1.40 & 0.64 & -- & -- & -- & -- \\
\hline trans - Cariofileno & -- & 2.53 & 1.39 & 1.31 & 1.12 & 0.98 & -- & 0.72 & 1.19 & 0.93 & 1.22 & 1.22 \\
\hline a-Humulene & -- & 0.36 & -- & 0.06 & 0.06 & -- & 0.13 & -- & 0.06 & -- & -- & -- \\
\hline Germacrene D & -- & 0.24 & -- & 0.13 & 0.14 & -- & -- & 0.14 & 0.05 & -- & 0.20 & 0.15 \\
\hline gama-Cadinene & 0.59 & 2.11 & 1.16 & 0.75 & 0.61 & 0.58 & 0.31 & 1.07 & 1.10 & 0.59 & 0.76 & 0.80 \\
\hline delta-cadinene & 0.09 & 0.35 & 0.26 & 0.17 & 0.17 & 0.14 & -- & 0.29 & 0.27 & 0.07 & 0.26 & 0.19 \\
\hline Citonelyl-n-Butirato & -- & 0.12 & -- & -- & -- & -- & 0.06 & -- & -- & -- & -- & -- \\
\hline Elemol & 0.05 & 0.37 & 0.16 & 0.09 & 0.18 & 0.14 & 0.08 & 0.11 & 0.18 & 0.07 & 0.15 & 0.13 \\
\hline Geranil Butirato & -- & 0.20 & 0.17 & -- & -- & -- & -- & 0.41 & 0.47 & 0.06 & -- & 0.14 \\
\hline Oxido Cariofileno & 0.42 & 0.68 & 0.85 & 0.51 & 0.55 & 0.54 & 0.69 & 0.90 & 0.88 & 0.06 & 0.08 & 0.14 \\
\hline Viridiflorol & 0.14 & 0.38 & -- & 0.07 & 0.15 & 0.13 & 0.32 & 0.35 & 0.16 & 0.06 & 0.28 & 0.18 \\
\hline a-Cadinol & 0.39 & 0.49 & 0.17 & 0.23 & 0.25 & 0.31 & 0.39 & 0.13 & 0.45 & 0.08 & -- & -- \\
\hline Decahidronaftaleno & 0.30 & 0.59 & 1.41 & 0.82 & 0.33 & 0.43 & -- & -- & 0.30 & 0.05 & 0.17 & 0.09 \\
\hline Farnesol & 5.50 & 5.80 & 5.29 & 2.25 & 2.41 & 1.92 & 10.74 & 4.50 & 1.41 & 1.50 & 1.66 & 1.72 \\
\hline cis-Nerolidol & 0.74 & -- & -- & -- & -- & -- & -- & -- & -- & -- & -- & -- \\
\hline trans - Nerolidol & -- & 0.31 & -- & -- & -- & -- & -- & -- & -- & -- & -- & -- \\
\hline Verbenol & 0.23 & -- & -- & -- & -- & -- & -- & -- & -- & -- & -- & -- \\
\hline Isoamyl & 0.53 & -- & -- & -- & -- & -- & -- & -- & -- & -- & -- & -- \\
\hline
\end{tabular}

* Means in each column followed by the same letter are not significantly different $(\mathrm{P}<0.05)$. 
Table 4. Composition of volatile oil from dry leaves of Java citronella (C. winterianus). Relative percentages as a function of seasons and harvest times.

\begin{tabular}{|c|c|c|c|c|c|c|c|c|c|c|c|c|}
\hline \multirow{2}{*}{ Compounds } & \multicolumn{3}{|c|}{ Summer } & \multicolumn{3}{|c|}{ Fall } & \multicolumn{3}{|c|}{ Winter } & \multicolumn{3}{|c|}{ Spring } \\
\hline & $9: 00 \mathrm{~h}$ & $12: 00 \mathrm{~h}$ & $15: 00 \mathrm{~h}$ & $9: 00 \mathrm{~h}$ & $12: 00 \mathrm{~h}$ & $15: 00 \mathrm{~h}$ & $9: 00 \mathrm{~h}$ & $12: 00 \mathrm{~h}$ & $15: 00 \mathrm{~h}$ & $9: 00 \mathrm{~h}$ & $12: 00 \mathrm{~h}$ & $15: 00 \mathrm{~h}$ \\
\hline & \multicolumn{12}{|c|}{ Relative percentage of chemical constituents } \\
\hline 6-metil-2-hepten-2-one & -- & -- & -- & -- & -- & -- & -- & 0.14 & -- & -- & -- & -- \\
\hline$\beta$-Mirceno & -- & -- & -- & -- & -- & -- & -- & 0.04 & -- & -- & -- & -- \\
\hline Octanal & -- & -- & -- & -- & -- & -- & -- & -- & -- & -- & -- & -- \\
\hline Limoneno & 2.79 & 2.79 & 2.39 & 1.14 & 1.18 & 1.01 & 2.28 & 2.58 & 2.22 & 1.91 & 1.70 & 1.43 \\
\hline$\alpha$-pinene & 0.24 & 0.23 & 0.23 & -- & -- & 0.08 & 0.22 & 0.27 & 0.12 & 0.17 & 0.10 & 0.08 \\
\hline Trans- $\beta$-Ocimene & -- & 0.04 & -- & -- & -- & -- & 0.09 & 0.12 & -- & -- & -- & -- \\
\hline Bergamal & 0.89 & 0.88 & 0.74 & -- & 0.13 & 0.16 & 0.75 & 0.59 & 0.30 & -- & -- & -- \\
\hline Nonanone & 0.42 & 0.47 & 0.43 & -- & -- & 0.09 & 0.44 & 0.50 & 0.18 & -- & 0.07 & -- \\
\hline Linalol & 0.99 & 1.48 & 1.37 & 0.65 & 0.45 & 0.74 & 1.08 & 1.59 & 0.89 & 0.96 & 0.84 & 0.83 \\
\hline Rose Oxide & 0.10 & 0.19 & 0.05 & -- & -- & -- & 0.05 & 0.10 & 0.04 & -- & -- & -- \\
\hline Isopulegol & 0.76 & 0.38 & 0.53 & 0.32 & 0.33 & 0.64 & 0.45 & 0.23 & 0.58 & 0.56 & 0.56 & 0.53 \\
\hline neo-Isopulegol & 0.24 & 0.56 & 0.77 & -- & -- & -- & 0.61 & 0.40 & 0.21 & -- & -- & -- \\
\hline Citronelal & 25.86 & 25.78 & 25.30 & 27.33 & 26.31 & 24.29 & 26.28 & 26.22 & 21.73 & 28.51 & 28.63 & 27.72 \\
\hline Terpenol-4 & 0.24 & -- & -- & -- & -- & -- & -- & -- & -- & -- & -- & -- \\
\hline cis-Decenal & 0.26 & -- & -- & -- & -- & -- & -- & -- & -- & -- & -- & -- \\
\hline Decanal & 0.65 & 0.67 & 0.66 & 0.23 & 0.24 & 0.45 & 0.73 & 0.75 & 0.60 & 0.42 & 0.31 & 0.31 \\
\hline Citronelol & 12.59 & 18.94 & 14.53 & 10.80 & 11.22 & 11.24 & 11.31 & 15.25 & 12.57 & 11.83 & 11.48 & 11.60 \\
\hline Neral & 6.18 & -- & 3.76 & 4.22 & 4.79 & 4.73 & 8.00 & 4.34 & 3.66 & 4.02 & 4.03 & 3.85 \\
\hline Geraniol & 34.35 & 35.22 & 37.55 & 43.89 & 44.31 & 48.82 & 36.93 & 34.47 & 42.34 & 42.04 & 42.75 & 43.00 \\
\hline Geranial & -- & -- & -- & 5.41 & 6.20 & -- & -- & -- & 1.60 & 4.64 & 3.44 & 4.54 \\
\hline Geranyl Formate & -- & 0.10 & 0.05 & -- & -- & -- & 0.06 & -- & -- & -- & -- & -- \\
\hline Citronelyl acetate & 0.57 & 0.45 & 0.76 & 0.76 & 0.22 & 0.94 & 0.90 & 1.36 & 1.27 & 0.21 & 0.69 & 0.85 \\
\hline Geranyl acetate & 0.92 & 0.95 & 1.24 & 1.48 & 1.09 & 2.04 & 1.73 & 2.46 & 2.68 & 1.58 & 1.66 & 2.35 \\
\hline$\beta$-Elemene & -- & -- & -- & -- & -- & -- & -- & -- & 0.17 & -- & -- & -- \\
\hline Tetradecanal & 0.29 & 0.35 & -- & -- & -- & -- & -- & -- & -- & -- & -- & -- \\
\hline trans - Cariofileno & 0.76 & 0.53 & 1.12 & 1.55 & 1.26 & 1.48 & 1.11 & 0.77 & 1.87 & 1.19 & 1.26 & 1.05 \\
\hline a-Humulene & -- & -- & -- & -- & -- & -- & -- & 0.07 & 0.18 & -- & 0.07 & -- \\
\hline Germacrene D & -- & -- & -- & -- & 0.10 & 0.19 & -- & -- & 0.17 & -- & 0.09 & 0.08 \\
\hline gama-Cadinene & 0.86 & 0.67 & 1.17 & 1.07 & 0.85 & 0.91 & 1.17 & 0.49 & 1.45 & 0.87 & 0.85 & 0.65 \\
\hline delta-cadinene & 0.17 & 0.13 & 0.24 & 0.11 & 0.10 & 0.31 & 0.24 & 0.13 & 0.36 & 0.09 & 0.11 & 0.10 \\
\hline Citonelyl-n-Butirato & -- & -- & -- & -- & -- & -- & 0.04 & -- & -- & -- & -- & -- \\
\hline Elemol & 0.06 & 0.05 & 0.12 & -- & -- & 0.09 & -- & 0.06 & 0.31 & -- & 0.08 & -- \\
\hline Geranil Butirato & 0.34 & 0.26 & 0.15 & -- & -- & -- & 0.29 & 0.29 & 0.60 & -- & 0.08 & -- \\
\hline Oxido Cariofileno & 1.14 & 1.05 & 0.99 & 0.44 & 0.66 & 0.42 & 0.95 & 0.58 & 1.06 & 0.10 & 0.10 & 0.21 \\
\hline Viridiflorol & -- & -- & -- & -- & -- & -- & -- & 0.04 & 0.34 & -- & 0.07 & 0.08 \\
\hline a-Cadinol & 0.12 & -- & 0.05 & 0.23 & 0.10 & 0.35 & -- & 0.11 & 0.66 & -- & 0.11 & -- \\
\hline Decahidronaftaleno & 0.27 & 0.10 & 0.66 & -- & -- & 0.09 & 0.28 & 0.11 & 0.07 & -- & 0.10 & -- \\
\hline Farnesol & 5.69 & 6.13 & 4.09 & 0.36 & 0.45 & 0.54 & 3.79 & 5.17 & 0.81 & 0.89 & 0.83 & 0.73 \\
\hline cis-Nerolidol & 0.31 & 0.37 & -- & -- & -- & -- & -- & -- & -- & -- & -- & -- \\
\hline trans - Nerolidol & -- & -- & -- & -- & -- & -- & -- & -- & -- & -- & -- & -- \\
\hline Verbenol & -- & -- & -- & -- & -- & -- & -- & -- & -- & -- & -- & -- \\
\hline Isoamyl & -- & -- & -- & -- & -- & -- & -- & -- & -- & -- & -- & -- \\
\hline
\end{tabular}

* Means followed by the same minuscule letter in each column and by the same capital letter in each line are not significantly different $(\mathrm{P}<0.05)$. 
Java citronella shows lower volatile oil yield during winter. Morning harvest provide higher yield. Chemical composition is affected by season and drying. However, no influence by time of harvest was observed on chemical composition.

\section{ACKNOWLEDGMENTS}

The authors wish to acknowledge ETENE/ FUNDECI/BNB for financial support and $\mathrm{CNPq}$ for supporting grants.

\section{REFERENCES}

Adams RP 1995. Identification of essential oil components by gas chromatograpy/mass spectroscopy. Ilinois: USDA - Allured Publishing Corporation.

American Spice Trade Association 1985. Official analytical methods of the American Spice Trade Association, 3 $e d$. The Association, Englewood Cliffs.

Arrigoni-Blank MF, Silva-Mann R, Campos DA, Silva PA, Antoniolli AR, Caetano LC, Sant'Ana AEG, Blank AF 2005. Morphological, agronomical and pharmacological characterization of Hyptis pectinata (L.) Poit germplasm. Rev Bras Farmacogn 15: 298-303.

Barbosa-Filho JM, Vasconcelos THC, Alencar AA, Batista LM, Oliveira RAG, Guedes DN, Falcão HS, Moura MD, Diniz MFFM, Modesto-Filho J 2005. Plants and their active constituents from South, Central, and North America with hypoglycemic activity. Rev Bras Farmacogn 15: 392-413.

Blank AF, Fontes SM, Carvalho-Filho JLS, Alves PB, SilvaMann R, Mendonça MC, Arrigoni-Blank MF, Rodrigues MO 2005. Influência do horário de colheita e secagem de folhas no óleo essencial de melissa (Melissa officinalis L.) cultivada em dois ambientes. Rev Bras Plantas Med 8: 73-78.

British Pharmacopoeia 1988. British Pharmacopoeia, 14 ed. Stationery Office, London.

Carvalho-Filho JLS, Blank AF, Alves PB, Ehlert PAD, Melo AS, Cavalcanti SCH, Arrigoni-Blank MF, Silva-Mann $\mathrm{R}$ 2006. Influence of the harvesting time, temperature and drying period on basil (Ocimum basilicum L.) essential oil. Rev Bras Farmacogn 16: 24-30.

Fatima S, Farooqi AHA, Sharma S 2002. Physiological and metabolic responses of different genotypes of Cymbopogon martinii and C. winterianus to water stress. Plant Growth Regul 37: 143-149.

Guenther E 1992. The essential oils. D. Van Nostrand, Princeton.

Innecco R, Cruz GF, Vieira AV, Mattos SH, Chaves CM 2003. Spacing, cut time and harvest number in Mentha $\mathrm{x}$ villosa Huds. Cienc Agron 34: 247-251.

Lorenzi H, Matos FJA 2003. Plantas medicinais no Brasil: nativas e exóticas. Plantarum, Nova Odessa, São Paulo.

Matos FJA 2000. Plantas medicinais - Guia de seleção e emprego de plantas medicinais do Nordeste do Brasil, 2 ed. Editora UFC, Fortaleza.

Morais SM, Dantas JDP, Silva ARA, Magalhães EF 2005.
Plantas medicinais usadas pelos índios Tapebas do Ceará. Rev Bras Farmacogn 15: 169-177.

Nagao EO, Innecco R, Mattos SH, Filho SM, Marco CA 2004. Effect of the harvest time on content and major constituent of essential oil of Lippia alba (Mill) N.E.Br., chemotype citral-limonene. Cienc Agron 35: 355-360.

Nascimento IB, Innecco R, Marco CA, Mattos SH, Nagao EO 2003. Efeito do horário de corte no óleo essencial de capim-santo. Cienc Agron 34: 169-172.

Pansera MR, Santos ACA, Paese K, Wasum R, Rossato M, Rota LD, Pauletti GF, Serafini LA 2003. Análise de taninos totais em plantas aromáticas e medicinais cultivadas no Nordeste do Rio Grande do Sul. Rev Bras Farmacogn 13: 17-22.

Rocha MFA, Borges NSS, Innecco R, Mattos SH, Nagao EO 2002. Influência do horário de corte sobre o citronelal do óleo essencial (Cymbopogon winterianus). Hortic Bras 20(Supl.): 1-4.

Sarma TC, Sharma RK, Adhikari RK, Saha BN 2001. Effect of altitude and age on herb yield, oil and its major constituents of Java citronella (Cymbopogon winterianus Jowitt.). J Essent Oil Bear Plants 4: 77-81.

Sarma TC 2002. Variation in oil and its major constituents due to season and stage of the crop in Java citronella (Cymbopogon winterianus Jowitt.). J Spices Arom Crops 11: 97-100.

Singi G, Damasceno DD, D’Andréa ED, Silva GAE 2005. Efeitos agudos dos extratos hidroalcoólicos do alho (Allium sativum L.) e do capim-limão (Cymbopogon citratus (DC) Stapf) sobre a pressão arterial média de ratos anestesiados. Rev Bras Farmacogn 15: 94-97.

Tôrres AR, Oliveira RAG, Diniz MFFM, Araújo EC 2005. Estudo sobre o uso de plantas medicinais em crianças hospitalizadas da cidade de João Pessoa: riscos e benefícios. Rev Bras Farmacogn 15: 373-380.

Vendruscolo GS, Rates SMK, Mentz LA 2005. Dados químicos e farmacológicos sobre as plantas utilizadas como medicinais pela comunidade do bairro Ponta Grossa, Porto Alegre, Rio Grande do Sul. Rev Bras Farmacogn 15: 361-372. 